\title{
Production and control of auditory signals in attention research using the Apple-Psych system
}

\author{
ROBERT S. BURKE \\ South Dakota State University, Brookings, South Dakota
}

\begin{abstract}
A technical issue in auditory spatial attention research is how best to provide low-cost, accurate control of the frequency, duration, and location of acoustic signals. Three solutions to the problem are compared: use of the internal oscillator of the Apple IIe, use of a Mountain A/D+D/A card, and use of an external oscillator chip. The unique advantages and drawbacks of each system are discussed. All share some common problems of external speaker interface, timing control, and interface with the software. Hardware and software solutions implemented on the Apple-Psych system are discussed, along with data that demonstrate the precision control achieved with the system.
\end{abstract}

Although many experiments in psychology use the computer as a stimulus presentation and control device for visual and verbal stimuli (Dorfman, 1987), there is also a need for solutions to the problems faced by researchers in the auditory laboratory. The typical issues faced in establishing a computer system are computer selection, system configuration, software control, stimulus generation, and interface design. This paper focuses on the use of the Apple Ile computer; the Apple-Psych software system, a Pascal-based software system for experimental control (Osgood, 1984a, 1984b); and three methods of producing auditory signals.

To be useful to the auditory researcher, a system must be relatively easy to use, must allow adequate control of signal properties, and should be relatively low cost. Manufacturers currently market precision oscillators with GPIB interfaces that allow effective computer control of auditory signal parameters, but such systems start in the range of $\$ 2,000$. For the typical laboratory, or for the student laboratory, such equipment is not generally feasible. However, there are suitable alternatives.

To facilitate the analysis of auditory research requirements, consider a three-level classification scheme for psychological experiments that use auditory stimuli. The classification depends on task requirements and the complexity of the signal. In level one experiments, auditory signals serve as simple cues. Typically, the researcher is interested in the timing and the duration (measured in 10 ths of a second) of the auditory signal, is content to use an unmodulated pure tone, uses audible levels of tones, and does not expect the central processor to be doing much else during the brief presentation of the stimulus. In level two experiments, the experimenter is in-

Funding for this research came in part from the Research Support Fund of South Dakota State University. I wish to extend special thanks to Amy Frick for programming support and to Warren Wieger for technical design of the audio oscillator and controller. Reprints are available from the author at the Department of Psychology, Box 504, South Dakota State University, Brookings, SD 57007. terested in more precise control of frequency and intensity, and timing precision is measured in milliseconds. The properties of the auditory stimuli, particularly the pitch, intensity, and duration of the tones, are the focus of the study. The solutions offered in this presentation primarily address level two stimulus configurations. Level three experiments are those in which modulation is required or complex tones are generated. Timing precision is measured in microseconds, and frequency and intensity require precision, on-line control. Although solutions for the level three experiments are not the primary focus of this discussion, there are solutions to the stimulus control and production issues inherent in the designs of the second and third options.

\section{Three Methods of Generation}

The first approach to sound generation is to use the computer itself. Newer computers, such as the Amiga and the Apple IIgs, have rich potential for the auditory researcher. Their multiple oscillator formats allow a wide range of signal possibilities. The Apple IIe has its own oscillator and internal speaker that can be used for level one research.

The Apple-Psych system contains a Pascal procedure called APPLESPEAK. The syntax of the statement is: APPLESPEAK (CYCLES, ONTIME, OFFTIME). The integer value for CYCLES can range from 1 to 255 , but exact frequency depends on the computer. The doubleprecision timing variables are passed to another procedure, WATCHWORLD, which manages an event queue. The APPLESPEAK procedure provides a simple way to control the frequency and duration of tones used in level one research. If an external speaker is desired or if intensity control is desired, it is a rather simple matter to redirect the output of the internal speaker to an amplifier and speaker system.

The advantage of this approach is its simplicity, but it has some obvious drawbacks if precision control of frequency and other sound characteristics are desired. It also 
does not allow other processing to occur while the speaker switch is being toggled by the CPU.

Method two is to use an auxiliary $A / D+D / A$ card. We used the $A / D+D / A$ card produced by Mountain Computer. This approach enables the computer to produce a digital representation of the analog signal, store the digits as a data set in an assembly language routine, and then call the assembly procedure through the Pascal code.

Our procedure was to generate a sine-wave signal of known frequency and use the FASTSAMPLE subroutine provided with the board to store a set of digitized values for the particular frequency. The Mountain Computer card has 16 channels of analog input and 16 channels of ana$\log$ output. It accepts voltage ranges from -5 to $+5 \mathrm{~V}$ and converts the voltage to a digital value in $9 \mathrm{msec}$. With the FASTSAMPLE program, there is a $50-\mathrm{kHz}$ sampling rate, theoretically sufficient to sample the frequencies up to $25 \mathrm{kHz}$. A BASIC routine was used to PEEK the memory locations and write the values to the screen.

To generate sounds through the D/A channels first required the development of an assembly language program (shown in Listing 1) to control interrupts, read and store appropriate return addresses and register values, read a series of digits stored as data in the program, and push these to the channel address of the D/A channel.

The Apple Pascal system has the ability to utilize external assembly language procedures that have been stored in the system library or that are defined as external procedures and assembled and linked to the specific program. We chose the latter option. In the Pascal main program, the procedure is invoked with a normal procedure call (in this example, PROCEDURE P1000;), which produces a tone of a specific frequency ( $1000 \mathrm{~Hz}$ in this example). In the current implementation, a separate machine language program has been used for each frequency desired in the experiment.

The advantage that the $\mathrm{A} / \mathrm{D}+\mathrm{D} / \mathrm{A}$ approach has over the internal oscillator is that there is increased precision in the control of the frequency and shape of the stimulus tone. Onset and offset times are controlled by the ApplePsych system. However, an external amplifier is still required to manipulate intensity levels. The approach also

LISTING 1

The 6502 Assembly Language Procedure to Generate a 1000-Hz Tone Through Channel 1 of the Mountain A/D $+D / A$ Card

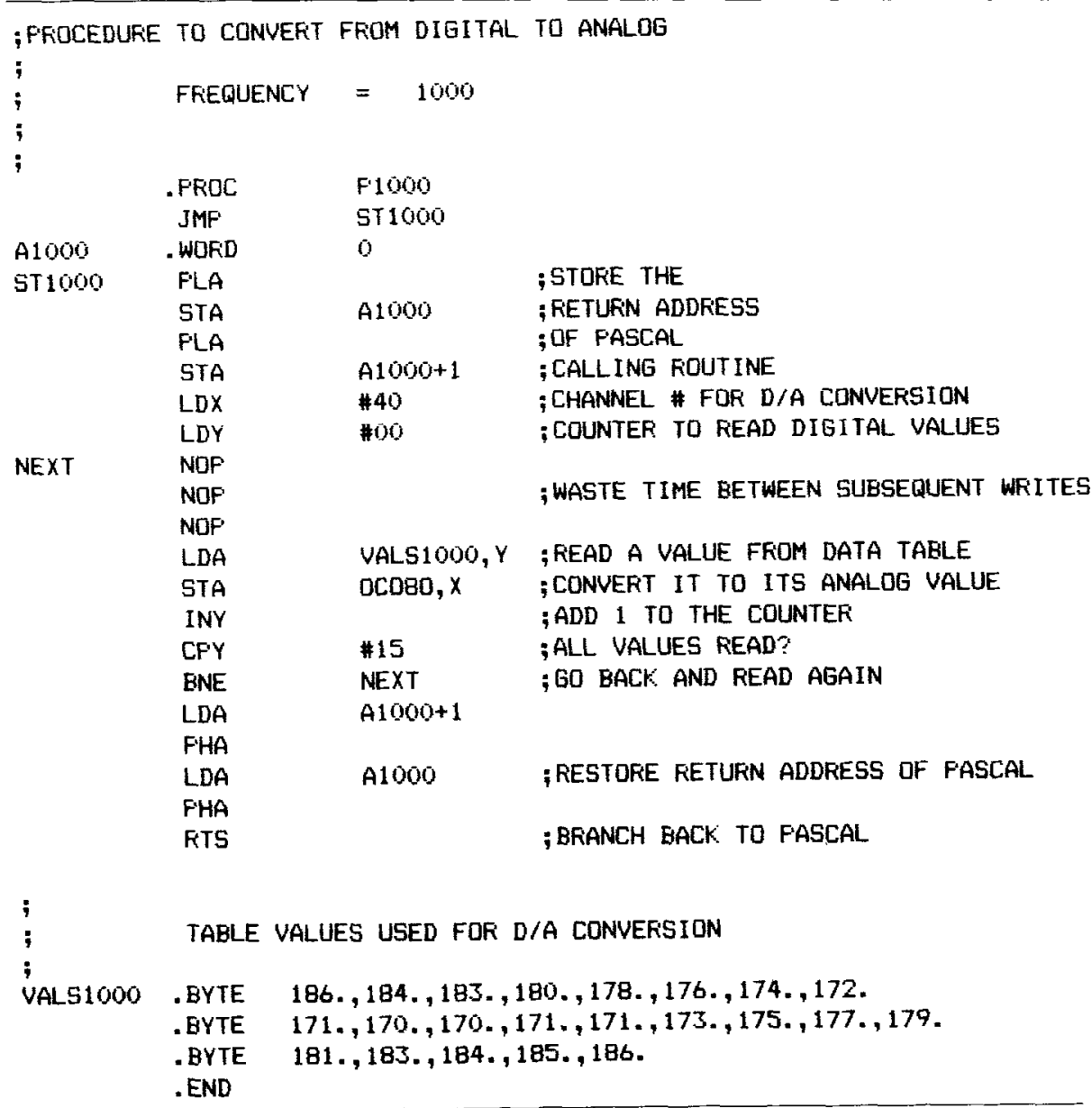


offers the potential to control the sound envelope and aspects of its complexity. Overall, it would be suitable for most level two research.

There are some disadvantages to the approach. The sampling rates form an upper bound to choice of frequency and modulation. The approach also requires that the central processor is consumed with the task of presenting the stimuli until an interrupt occurs or until the time of the event is over. Although this is not an insurmountable problem, it requires careful programming in an on-line environment.

Method three requires an external oscillator that is under computer control. A number of sound cards are available for the Apple, none of which were used for the present project. They were not chosen because of cost considerations, stimulus control and timing considerations, and the need for some kind of external switching interface. Auxiliary oscillator cards may be the solution of choice for other machine systems.

Our solution, diagrammed in Figure 1, was to use a quad op-amp chip configured as a sine-wave function generator, whose frequency was controlled by a set of switchable resistance values. The output of the chip was then directed to one of eight amplifier channels, each one attached to a 12.7-cm speaker (Radio Shack model no. 401909B) with a frequency response range of $70-15000 \mathrm{~Hz}$. The outputs of each channel were individually adjusted to achieve physical and subjective balance across channels. The hardware control of each channel was accomplished through a parallel interface I/O card (California Computer Systems), which provides TTL logic levels to the audio interface card. The output of the oscillator was calibrated against the output of an audio oscillator.
Control of the external oscillator is accomplished through the Pascal program and the $\mathrm{I} / \mathrm{O}$ and timing routines of the Apple-Psych system invoked by the procedure PUTSW. This procedure passes three variables, a device selection parameter, the subset of switches to change, and the time at which the switching is to take place.

Method three offers some advantages of relatively lowcost interfacing and auditory frequency control. The solution is not particularly novel, but it does demonstrate the ease with which the Apple-Psych system can be integrated with external devices. The current disadvantage of the system is lack of real-time control of signal intensity or pitch. Future models of the interface will use the I/O card and the analog control channels of the A/D converter for this control.

\section{A Research Example}

To demonstrate the capabilities of the Apple-Psych system with the interface described in method three, I describe below one experiment from a series on auditory spatial attention. The experiment was performed to empirically determine the effectiveness of a brief sequence of three tones in producing the impression of directionality. The specific goal was to establish an auditory analog of an arrow used in visual spatial attention studies (Posner, Snyder, \& Davidson, 1980). Once a suitable "auditory arrow" was established, it could then be used in other attention research procedures.

Twelve volunteer college students participated in a 45min session. Their basic task was to discriminate the directionality of a sequence of three sounds and respond by pressing a left or a right key. Pure tones of $730 \mathrm{~Hz}$ were

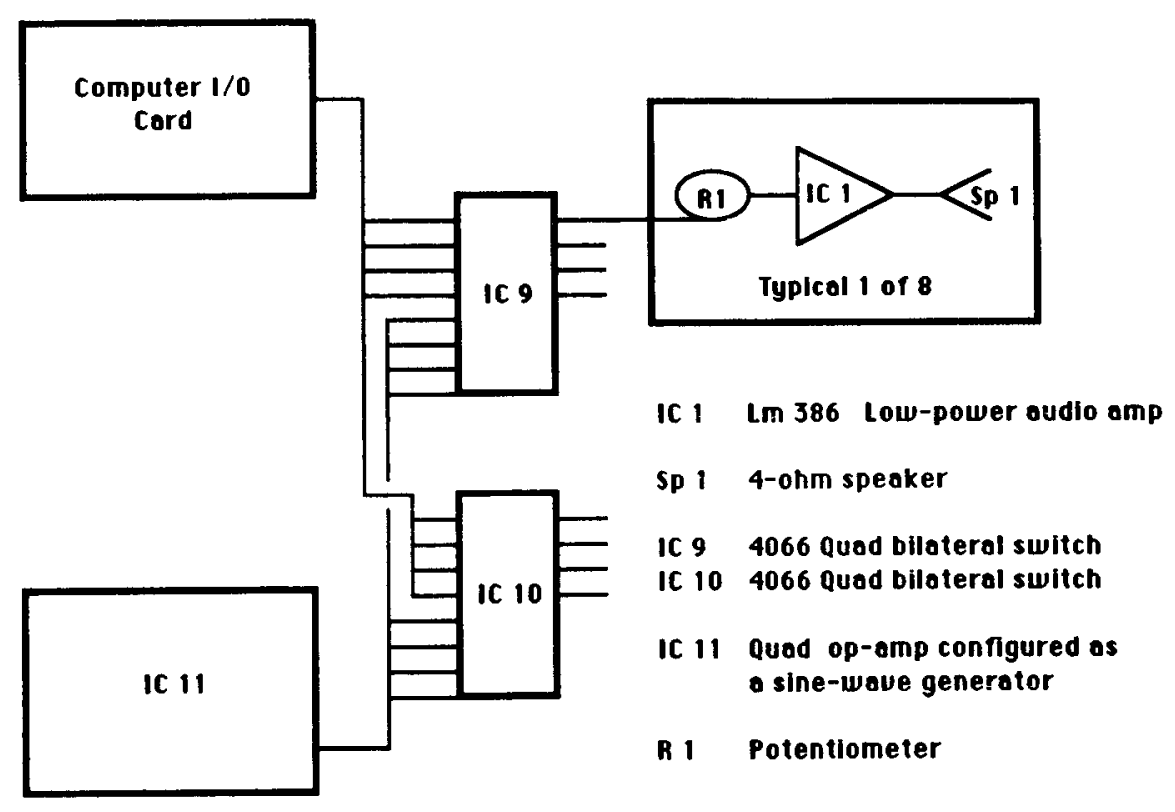

Figure 1. A schematic diagram of the external audio oscillator and computer control channels through the California Computer System parallel I/O card. 
presented at $72 \mathrm{~dB}$ SPL through three speakers mounted immediately in front of the subject. The speakers were centered at $0^{\circ}$ and at $10^{\circ}$ left and right of center and were mounted on a semicircular rack with a $1 \mathrm{~m}$ radius from the center of the head restraint device.

The subjects heard equal numbers of tone series that "swept" left, "swept" right, or stayed in the center. They were told not to respond to center tones, but to respond as quickly and accurately as they could to the "sweeping" series. Auditory sequences lasted 100, 200, or $300 \mathrm{msec}$. The sounds also had either a $10 \%-, 50 \%-$, or $100 \%$-on duty cycle. The subjects received nine blocks of 54 trials, one block per sequence length and duty cycle combination.

This particular experiment demonstrates the precision control possible with the Apple Psych system. For example, in the 100 -msec signal each speaker was on for $33 \mathrm{msec}$ in the $100 \%$-on duty cycle and was on for $3 \mathrm{msec}$ in the $10 \%$-on duty cycle.

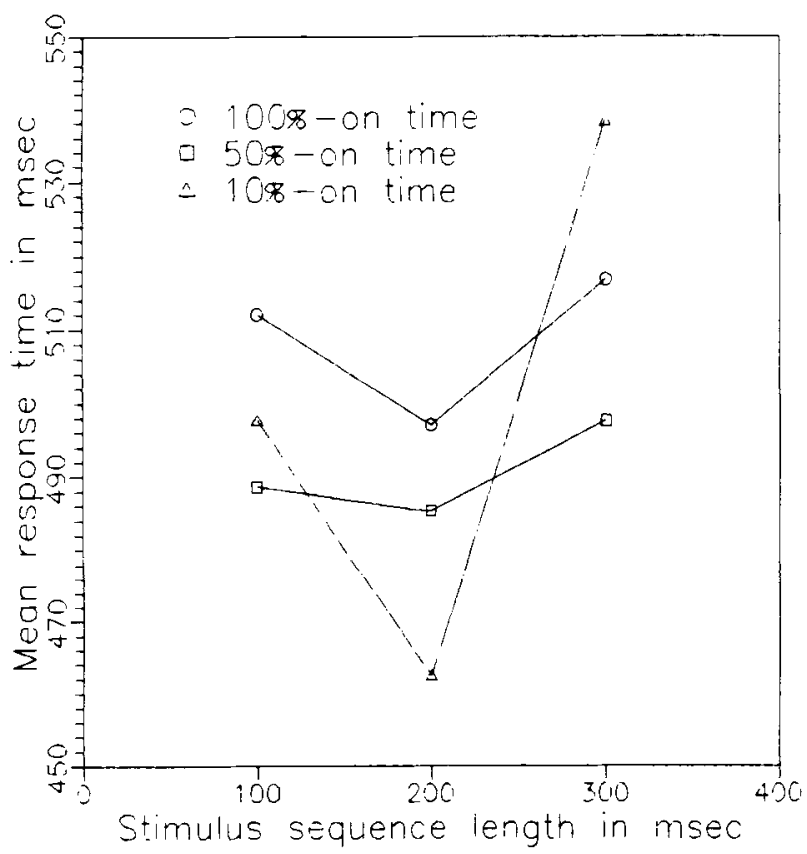

Figure 2. Mean response times to directional sequences of tones varying in length and percent-on time.
Figure 2 presents the reaction time data, which suggest that the subjects responded most quickly to stimuli lasting $200 \mathrm{msec}$ under the $10 \%$-on duty cycle. The leading edge of each tone seems to have been the critical information (Hafter \& Buell, 1985). This condition produced slightly more errors (less than $1 \%$ ) but significantly $(p<.01)$ faster reaction times than the comparable 300 -msec condition. This is taken as evidence that subjects are able to develop an impression of directionality from exposures to brief sequences of auditory stimuli.

\section{Conclusion}

There is a continuing need for researchers in psychology laboratories to develop interface techniques for stimulus presentation and response collection. This paper has presented three ways in which auditory stimuli can be produced with the Apple II series of computers using the Apple-Psych system for software control. It is evident that there is some flexibility in the way auditory signals are generated and that the choice of procedure depends on the level of precision required by the experiment. For level one experiments, method one is acceptable and requires a minimum of interface techniques. For level two research, the second and third methods described provide adequate solutions, depending on the goal of the researcher. It was not within the scope of this paper to address solutions for level three research; however, methods two and three, with refinement, may provide beginning solutions.

\section{REFERENCES}

DoRFMAN, D. (1987). Software timing of events in cognitive psychology experiments. Behavior Research Methods, Instruments, \& Computers, 19, 185-190.

HAFTer, E. R., Buell, T. N. (1985). The importance of transients for maintaining the separation of signals in auditory space. In M. I. Posner \& D. S. M. Marin (Eds.), Attention and Performance XI (pp. 337-354). Hillsdale, NJ: Erlbaum.

OsGOOD, G. (1984a). Hardware implementation and utility manual (Coglab \#7). Eugene, OR: University of Oregon, Psychology Department.

OsGooD, G. (1984b). Software program development guide (Coglab \#8). Eugene, OR: University of Oregon, Psychology Department.

Posner, M. I., SNyder, C. R. R., \& Davidson, B. J. (1980). Attention and the detection of signals. Journal of Experimental Psychology: General, 109, 160-174. 